\title{
SOFTWARE EDUCATIVO SOBRE DIABETES MELLITUS PARA PROFISSIONAIS DE SAÚdE: ETAPAS DE ELABORAÇÃO E DESENVOLVIMENTO ${ }^{1}$
}

Erika Christiane Marocco Duran ${ }^{2}$

Maria Inês Monteiro Cocco ${ }^{3}$

Duran ECM, Cocco MIM. Software educativo sobre diabetes mellitus para profissionais de saúde: etapas de elaboração e desenvolvimento. Rev Latino-am Enfermagem 2003 janeiro-fevereiro; 11(1):104-7.

Esta pesquisa tem por objetivo elaborar um software sobre diabetes mellitus para dar subsídios à educação continuada dos profissionais de saúde e suas práticas educativas. O sistema foi desenvolvido utilizando o aplicativo Delphi, que manipula banco de dados, usando palavras-chave para sua abertura e utilizando a linguagem de programação Pascal. O software inclui mecanismos de busca, por meio de palavras-chave, agilizando o processo de consulta da base de dados.

DESCRITORES: software, diabetes mellitus, educação continuada, educação em saúde, enfermagem

\section{EDUCATIONAL SOFTWARE ABOUT DIABETES MELLITUS TO HEALTH PROFESSIONALS: ELABORATION AND DEVELOPMENT STAGES}

This research aimed at creating a software about Diabetes mellitus in order to stimulate the continuing education of health professionals as well as educative practices. The system was developed using Delphi application, through a data bank utilizing key-words and the Pascal language. The software includes key-words searching tools, improving the process of accessing the data bank.

DESCRIPTORS: software, diabetes mellitus, continuing education, education and health, nursing

\section{SOFTWARE EDUCATIVO SOBRE DIABETES MELLITUS PARA PROFESIONALES DE SALUD: ETAPAS DE ELABORACIÓN Y DESARROLLO}

Esta investigación tiene por objetivo elaborar un software sobre diabetes mellitus para dar aportes a la educación continuada de los profesionales de salud en su prácticas educativas. El sistema fue desarrollado utilizando el aplicativo Delphi, que manipula banco de datos usando palabras llaves para su apertura y utiliza el lenguaje de programación Pascal. El software incluye mecanismos para búsqueda, usando palabras llave, dando agilidad para el proceso de consulta da base de datos.

DESCRIPTORES: software, diabetes mellitus, educación continuada, educación en salud, enfermería

\footnotetext{
${ }^{1}$ Pesquisa de iniciação científica financiada através do Programa PIBIC-CNPq; ${ }^{2}$ Enfermeira, Licenciada em Enfermagem, Mestre em Enfermagem;

${ }^{3}$ Enfermeira, Professor Doutor, e-mail: inesmon@obelix.unicamp.br; Membro do Grupo de Estudos e Pesquisas em Saúde e Trabalho, do Departamento de Enfermagem e Membro do PRAESA - Laboratório de Práticas Educativas em Saúde. Faculdade de Ciências Médicas da Universidade Estadual de Campinas
} 
INTRODUÇÃO

$\boldsymbol{O}$ diabetes mellitus tem, atualmente, alta prevalência no Brasil e no mundo, sendo fundamental, para seu tratamento e a prevenção das complicações, que os profissionais de saúde e os clientes diabéticos e seus familiares tenham acesso a informações sobre a doença.

Os profissionais de saúde e o enfermeiro, em particular, têm um papel importante no que se refere às práticas educativas em saúde, que são atividades essenciais, presentes em seu trabalho cotidiano.

"As práticas educativas em saúde têm estado presentes no cotidiano dos profissionais de saúde e dos usuários dos serviços de saúde, porém, não têm conseguido, via de regra, gerar grandes transformações, que tenham impacto no modo de vida da população, em suas condições de saúde e na construção de sua cidadania"(1).

Deve-se, ainda, salientar que o processo educativo deve ser de qualidade, garantir uma relação significativa entre o profissional e a clientela, procurando, dessa forma, assistir, adequadamente, aos educandos, visando a um aprendizado satisfatório, assim como estimular o educador (profissional) de saúde acerca da utilização de práticas educativas condizentes com a realidade da clientela.

A informática em enfermagem é fundamental para o desenvolvimento profissional, pois pode auxiliar o enfermeiro na tomada de decisões clínicas, dentre outras possibilidades $^{(2)}$.

A educação continuada dos profissionais, técnicos e auxiliares que integram as equipes interdisciplinares de saúde, é fundamental para mantê-los atualizados e aperfeiçoar seus conhecimentos e habilidades.

$\mathrm{Na}$ revisão realizada para a elaboração do software, foram encontradas pesquisas sobre o uso de computador para a realização de práticas educativas, tanto para os profissionais de saúde, quanto para a clientela.

O Censo Nacional de Diabetes realizado no Brasil, em 1988, população urbana de nove capitais, na faixa etária de 30 a 69 anos, apontou uma prevalência de 7,6\%, com metade desse total desconhecendo a sua condição ${ }^{(3)}$.

Na Austrália, há 340.000 diabéticos conhecedores do diagnóstico e 250.000 que o desconhem. O Plano de Ação Nacional (NAP), realizado em 1993, focou o cuidado preventivo e o diagnóstico precoce do diabetes, sendo identificada a necessidade de preparar profissionais para reduzir a morbidade e a mortalidade do paciente diabético no futuro. Para isso, as ações de enfermagem direcionadas para o cliente diabético foram consideradas um dos elementos mais importantes ${ }^{(4)}$.

A inovação tecnológica, assim como o uso de computadores na educação em diabetes, pode ser conceituada como um processo, no qual ela é adaptada ao indivíduo todo o tempo. Fatores que auxiliem na explicação e entendimento da adoção de uma inovação tecnológica podem contribuir para entender as mudanças de comportamento decorrentes do diabetes ${ }^{(5)}$.

Lewis refere que Takabayashi desenvolveu um programa de computador, para educação de pacientes diabéticos que utilizavam insulina, os quais receberam informações básicas usando o computador. Isso levou o educador em diabetes a providenciar mais instruções individualizadas. A pesquisa mostrou que o computador na educação em diabetes auxiliava no processo de aprendizagem e reduzia o tempo de ensino para menos de $40 \%{ }^{(5)}$.

Em uma pesquisa desenvolvida por LEWIS (1996), a qual avaliou os programas de diabetes conduzidos pelo National Diabetes Information Clearinghouse, confirmouse a escassez de programas de computador para a educação de pacientes, pois $16 \%$ eram programas administrativos, $8 \%$ de educação para profissionais e $60 \%$ para assessorar os nutricionistas na análise dietética. Os resultados da pesquisa mostraram que os educadores tinham pequena experiência com o computador. Além disso, apontou também a receptividade dos educadores para com a tecnologia, por meio da busca de conhecimento, aceitando a nova estratégia educacional ${ }^{(5)}$.

Nos países industrializados, os profissionais de saúde estão alterando o plano de intervenções educacionais a fim de promover o desenvolvimento de habilidades para o autocuidado dos clientes, conduzindo à melhoria da saúde. Alguns problemas enfrentados pelo educador em saúde incluem a diversidade da população, as dificuldades de aprendizagem e de ensino e o decréscimo de recursos para a educação, que podem ser particularmente incrementados pelo uso do computador. Para os educadores em saúde, em países desenvolvidos, ocorreram importantes avanços tecnológicos no século $X X$, destacando-se o uso do computador na educação dos clientes, permitindo a participação interativa e a sua eficiência, promovendo o interesse e maior motivação no usuário $^{(5)}$.

Em estudo realizado com pacientes diabéticos, 
a utilização do computador na educação dos clientes foi mais eficaz na mudança do comportamento e costumes do que o aprendizado tradicional. No grupo que utilizava o computador, constatou-se um declínio no valor de glicose no sangue e um resultado positivo em relação à alteração de comportamento ${ }^{(5)}$.

Assim sendo e por acreditar que um programa de computador $^{(6)}$, direcionado aos profissionais de saúde, juntamente com a adoção de outras estratégias educativas, possa facilitar o desenvolvimento do processo educativo de clientes com diabetes mellitus, optou-se pelo desenvolvimento de um software sobre Diabetes mellitus, cujas etapas serão descritas neste trabalho.

\section{OBJETIVO}

Elaborar software sobre diabetes mellitus para subsidiar a educação continuada dos profissionais de saúde e às suas práticas educativas.

\section{MATERIAL E MÉTODOS}

Propôs-se, nesta pesquisa, elaborar um programa educacional de ensino continuado, por meio de softwares educativos, aos profissionais de saúde, por serem multiplicadores do saber.

Foi realizado um levantamento bibliográfico, nas seguintes bases de dados: MEDLINE, LILACS, UNIBIBLI, com a finalidade de identificar os dados essenciais para a montagem de um Programa de Educação em Diabetes para profissionais de saúde e para fundamentação quanto aos aspectos de informática.

O sistema foi desenvolvido em conjunto com profissionais da área de Engenharia de Computação e da área de Análise de Sistemas, utilizando o aplicativo Delphi, que manipula banco de dados, valendo-se de palavraschave para sua abertura e utilizando como linguagem de programação Pascal, nos Laboratórios de Informática da Universidade Estadual de Campinas-UNICAMP.

\section{ETAPAS DA ELABORAÇÃO DO SOFTWARE}

Revisão bibliográfica

A revisão bibliográfica foi realizada por meio de levantamento em bases de dados das Bibliotecas da Universidade Estadual de Campinas-UNICAMP e em biblioteca particular, tendo possibilitado o acesso às informações necessárias para a elaboração do software.

O levantamento foi realizado com a utilização do Medline, Lilacs, Sociedade Brasileira de Endocrinologia Internet, International Nursing Index, Index Medicus e Index Latino-americano.

Foram selecionados e impressos aproximadamente quinhentos artigos - com resumo - sendo realizada, posteriormente, a delimitação dos temas que mais se aproximaram do objeto de estudo. A partir desse levantamento foram consultados os periódicos nacionais e internacionais. O levantamento foi realizado no período de setembro a dezembro de 1998.

\section{Desenvolvimento do software}

Inicialmente foram definidos os itens a serem trabalhados no software. Baseando-se na literatura consultada e em pesquisa anterior ${ }^{(7)}$, realizada junto à clientela de diabéticos, em um hospital universitário, a abordagem foi direcionada aos seguintes tópicos: definição do diabetes do tipo 1 e tipo 2, complicações crônicas e agudas, atividade física, dietoterapia, cuidados com os pés, gestação, tratamento e aplicação de insulina.

Essa estrutura foi apresentada para análise dos profissionais de informática e seguiu as seguintes fases de desenvolvimento: levantamento de dados, modelagem dos dados, desenvolvimento do software com a participação dos autores e dos profissionais de informática.

Os tópicos citados anteriormente foram relacionados, primeiramente, em uma página de apresentação em forma de itens, sendo que cada um deles, quando acionados, conduzem a uma explicação detalhada (com textos e figuras ilustrativas), a fim de informar o profissional de saúde. Esta segunda página está "oculta" e somente é visualizada se o processo anterior for realizado.

O software inclui, também, mecanismos de busca, utilizando palavras-chave, agilizando o processo de consulta da base de dados. Digita-se, por exemplo, o termo hipoglicemia, em uma caixa de diálogo específica, fornecida pela página de apresentação, no menu, a palavrachave hipoglicemia, e o software busca, em seu banco de dados, as referências mais próximas sobre tal assunto.

Após a concretização, o software foi gravado em CD-ROM. 
Apresentação do software

O sistema consiste de uma tela principal, onde são visualizados todos os assuntos existentes.

Dentro dessa tela principal, aparecem as seguintes palavras:

PÂNCREAS, INSULINA, DIABETES MELLITUS, CUIDADOS COM OS PÉS E DIABETES E GRAVIDEZ.

Cada item conduz a telas secundárias, nas quais as informações podem ser obtidas. Entretanto, há algumas variantes. No caso dos itens DIABETES MELLITUS e DIABETES E GRAVIDEZ, ao clicar neles, aparecerá outra caixa para que o usuário escolha, dentro do assunto, a abordagem desejada.

Como exemplo, no item DIABETES E GRAVIDEZ, observa-se a caixa contendo HISTÓRICO, AVALIAÇÃO DA VITALIDADE FETAL, PRINCÍPIOS GERAIS DE SEGUIMENTOE TRATAMENTO (o qual também apresenta outra caixa), COMPLICAÇÕES CLÍNICAS E OBSTÉTRICAS, O PARTO e O RECÉM-NASCIDO.

Vale ressaltar que todos os itens que apresentam setas "ocultam" o conteúdo e, quando clicados, expõemnos. Os itens que, depois de acionados, têm como resultados figuras, devem ser clicados novamente no texto escrito sobre elas, com a finalidade de visualizar o conteúdo (o que acontece no seguinte caminho DIABETES MELLITUS - TRATAMENTO - INSULINA e DIABETES MELLITUS - COMPLICAÇÕES).

Em algumas telas, encontram-se, no final, além do botão VOLTAR (presente em todas, para se retornar à tela anterior), botões IMAGENS (PÂNCREAS), TABELAS (DIABETES MELLITUS - TRATAMENTO - HIPOGLICEMIA ORAL), FASES (DIABETES E GRAVIDEZ - PRINCÍPIOS GERAIS DE SEGUIMENTO E TRATAMENTO) e TABELA

\section{REFERÊNCIAS BIBLIOGRÁFICAS}

1. Cocco MIM. Práticas educativas em saúde e a construção do conhecimento emancipatório. In: Bagnato MHS, Cocco MIM, Sordi MRL, organizadoras. Educação, saúde e trabalho: antigos problemas, novos contextos, outros olhares. Campinas (SP): Alínea; 1999. p.63-70.

2.Nagelkerk J, Ritola PM, Vandort PJ. Nursing informatics: the trend of the future. J Cont Educ Nurs 1998; 29(1):17-21.

3. Ministério da Saúde (BR). Secretaria de Assistência à Saúde. Departamento de Assistência e Promoção à Saúde. Coordenação de Doenças Crônico-degenerativas. Manual de diabetes. $2^{\underline{a}}$ ed. Brasília: Ministério da Saúde; 1993.
(DIABETES E GRAVIDEZ - COMPLICAÇÕES CLÍNICAS E OBSTÉTRICAS).

No menu principal, além dos guias ARQUIVO e PESQUISAR, existe o SOBRE, que refere informações acerca dos autores e dos profissionais que desenvolveram o software.

\section{CONSIDERAÇÕES FINAIS}

O diabetes melittus é citado entre as dez maiores causas de óbito na maioria dos países. As mortes precoces, geralmente causadas pelas complicações relacionadas a essa enfermidade poderiam ser evitadas. Os diabéticos e seus familiares necessitam de conhecimentos satisfatórios sobre a doença visando ao tratamento adequado e à conseqüente prevenção das complicações, melhorando sua qualidade de vida.

A realização deste software sobre diabetes para profissionais de saúde visa dar subsídios para sua educação continuada, assim como para as práticas educativas realizadas por eles junto à clientela.

Desenvolvido em uma linguagem atual, o software contribui para que o conhecimento existente seja realmente eficaz, objetivando uma sensível melhora nas práticas educativas dos profissionais de saúde junto aos clientes diabéticos.

\section{AGRADECIMENTOS}

Agradecemos à Engenheira de Computação Renata Carla Marocco e ao Analista de Sistemas Allysson Flávio Melo de Oliveira, pelo desenvolvimento do sistema em conjunto com as autoras.

4. Dunning T. Development of a nursing care manual to improve the knowledge of nurses caring for hospitalized patients with diabetes. J Cont Educ Nurs 1995; 26(6):261-6. 5. Lewis D. Computer based point education used by diabetes educators. Diabetes Educator 1996; 22(2):140-5.

6. Prado MEBB. O uso do computador na formação do professor: um enfoque reflexivo da prática pedagógica. [Dissertação]. Campinas (SP): Faculdade de Educação/ UNICAMP; 1996.

7. Marocco EC, Cocco MIM. Práticas Educativas em diabetes mellitus: proposta para elaboração de material pedagógico. Campinas: Universidade Estadual de Campinas; 1997. 